\begin{tabular}{|l|}
\hline A\&A manuscript no. \\
(will be inserted by hand later) \\
\hline Your thesaurus codes are: \\
missing; you have not inserted them
\end{tabular}

ASTRONOMY

\title{
ROSAT observations of A0538-66 during quiescence
}

\author{
S. Campana ${ }^{1,2}$ \\ 1 Osservatorio Astronomico di Brera, Via Bianchi 46, I-22055 Merate (Lc), Italy; \\ e-mail: campana@merate.mi.astro.it \\ 2 Affiliated to I.C.R.A.
}

Received ; Accepted

\begin{abstract}
We discuss serendipitous ROSAT observations of the Be transient A0538-66, which contains the fastest known accreting $\mathrm{X}$-ray pulsar $(69 \mathrm{~ms})$. We show that this transient is detected several times during quiescence at a level of about $10^{34-35} \mathrm{erg} \mathrm{s}^{-1}$. Considering this low luminosity it is more likely that $\mathrm{X}$-rays are produced by matter falling onto the neutron star magnetosphere, rather than onto the star surface. This could be the first example of an X-ray source in the "propeller regime".
\end{abstract}

Key words: Stars: individual: A0538-66 - X-ray: binaries - accretion

\section{Introduction}

The X-ray transient A0538-66 in the Large Magellanic Cloud contains the accreting neutron star with the shortest known spin period $(69 \mathrm{~ms})$. The periodic recurrence of most of its X-ray outbursts is highly suggestive of a $16.7 \mathrm{~d}$ (White \& Carpenter 1978) eccentric orbit ( $e \gtrsim 0.4)$ around the Be star companion (Johnston, Griffiths \& Ward 1980; Skinner et al. 1982). While in outburst, peak X-ray luminosities of $\sim 10^{39} \mathrm{erg} \mathrm{s}^{-1}$ as well as $\sim 2$ mag enhancements of the optical counterpart have been detected (Skinner 1980). The outburst spectrum was collected by the Monitor Proportional Counter (MPC) onboard Einstein. The best fit was provided by a black body model with a temperature $T_{\mathrm{bb}} \sim 2.4 \mathrm{keV}$, an emitting radius $R_{\mathrm{bb}} \sim 10^{6} \mathrm{~cm}$ and a column density $N_{H} \sim 5 \times 10^{22} \mathrm{~cm}^{-2}$ (Ponman, Skinner \& Bedford 1984). The large emission radius makes unlikely that this radiation arises as black body emission from a hot spot on the neutron star surface; rather this component was interpreted as the emission of a hot shell of material outside the magnetospheric radius (Ponman et al. 1984).

During the ROSAT all-sky survey two weaker outbursts from A0538-66 were detected, with peak luminosities of $\sim 4$ and $\sim 2 \times 10^{37} \mathrm{erg} \mathrm{s}^{-1}$ in the $0.1-2.4 \mathrm{keV}$ range
(Mavromatakis \& Haberl 1993). The emission was well fitted by a black body spectrum with a softer temperature $T_{\mathrm{bb}} \sim 0.2 \mathrm{keV}$, a characteristic radius $R_{\mathrm{bb}} \lesssim 5 \times 10^{7} \mathrm{~cm}$ and a smaller column density $N_{H} \sim 10^{20} \mathrm{~cm}^{-2}$.

ASCA detected a similar outburst on Feb 3, 1995 at predicted periastron passage at a level of $\sim 5.5 \times$ $10^{36} \mathrm{erg} \mathrm{s}^{-1}$ (1-10 keV; Corbet et al. 1995; Corbet 1996). A $3 \sigma$ upper limit of $\sim 2 \times 10^{35} \mathrm{erg} \mathrm{s}^{-1}(0.15-4.5 \mathrm{keV})$ on the quiescent luminosity of A0538-66 was determined with the Imaging Proportional Counter onboard Einstein (Long, Helfand \& Grabelsky 1981).

Here we discuss 25 serendipitous ROSAT Position Sensitive Proportional Counter (PSPC) pointings containing A0538-66 in the field of view, mainly carried out during a Large Magellanic Cloud survey. During these observations A0538-66 was detected seven times and on two occasions the number of photons was sufficient to extract a meaningful spectrum (section 2). In section 3 we discuss these observations in light of the possibility that matter, accreting at such low rates, cannot reach the neutron star surface but it is stopped at the magnetosphere by the centrifugal force exerted by the corotating magnetic field (i.e. centrifugal inhibition of accretion; see Stella et al. 1986; Campana et al. 1995). Our conclusions are summarised in section 4.

\section{ROSAT pointings}

As part of an on-going program aimed at studying the quiescent emission of hard and soft X-ray transients, we select different public archive ROSAT observations of A0538-66 obtained with the PSPC during 1992 and 1994 (see Table 1). Most of these observations were carried out during a Large Magellanic Cloud survey and are characterized by very short exposure times. On one occasion a deep pointing was performed, having as a target $4 \mathrm{U} 0532$ 66. All these observations were not centered at A0538-66, resulting in off-axis angles varying between 4' and 41' (see Table 1). 
Table 1. Observations log.

\begin{tabular}{|c|c|c|c|c|}
\hline $\begin{array}{c}\text { Obs. ID. } \\
\text { (ROR) }\end{array}$ & Date & $\begin{array}{c}\text { Time } \\
(\mathrm{s})\end{array}$ & $\begin{array}{r}\text { Off-axis } \\
\text { (arcmin) }\end{array}$ & $\begin{array}{l}\text { Orbital } \\
\text { Phase* }^{*}\end{array}$ \\
\hline $\mathrm{rp} 400246$ & Jul 9, 1992 & 14521 & 34.2 & $0.62-0.65$ \\
\hline wp900538 & Jul 22, 1993 & 1377 & 22.8 & 0.36 \\
\hline wp900539 & Jul 23, 1993 & 1377 & 25.1 & 0.42 \\
\hline wp900549 & Jul 24, 1993 & 1328 & 11.2 & 0.43 \\
\hline summed & Jul 1993 & 4082 & & $0.36-0.43$ \\
\hline wp900543 & Oct 7,1993 & 1093 & 33.8 & 0.94 \\
\hline wp900532 & Nov 4, 1993 & 1244 & 19.2 & 0.61 \\
\hline wp900530 & Nov 28,1993 & 426 & 23.1 & 0.07 \\
\hline wp900537 & Nov 28, 1993 & 363 & 13.4 & 0.07 \\
\hline wp900531 & Nov 29, 1993 & 536 & 26.7 & 0.13 \\
\hline wp900544 & Nov 29, 1993 & 2121 & 30.1 & $0.13-0.17$ \\
\hline wp900545 & Nov 29, 1993 & 363 & 31.9 & 0.17 \\
\hline wp900534 & Nov 30, 1993 & 295 & 8.7 & 0.17 \\
\hline wp900551 & Dec 1, 1993 & 990 & 18.6 & 0.29 \\
\hline wp900540 & Dec 2, 1993 & 1005 & 37.8 & 0.35 \\
\hline wp900552 & Dec 3, 1993 & 1071 & 5.3 & 0.41 \\
\hline wp900553 & Dec 3, 1993 & 1071 & 26.4 & $\dagger$ \\
\hline wp900533 & Dec 6, 1993 & 1579 & 25.1 & 0.57 \\
\hline summed & Nov-Dec 1993 & 8749 & & $0.07-0.57$ \\
\hline$\overline{\text { wp900541 }}$ & Jun 5,1994 & 1002 & 39.1 & 0.46 \\
\hline wp900542 & Jun 5, 1994 & 1000 & 41.4 & 0.46 \\
\hline summed & Jun 1994 & 2002 & & 0.46 \\
\hline wp900550 & Jul 4, 1994 & 970 & $\overline{15.9}$ & 0.21 \\
\hline wp900535 & Jul 5, 1994 & 855 & 13.5 & 0.24 \\
\hline wp900536 & Jul 6, 1994 & 1006 & 8.4 & 0.30 \\
\hline wp900547 & Jul 6, 1994 & 774 & 18.6 & 0.30 \\
\hline wp900546 & Jul 6, 1994 & 596 & 15.5 & 0.31 \\
\hline wp900548 & Jul 6, 1994 & 1071 & 4.1 & 0.31 \\
\hline summed & Jul 1994 & 5272 & & $0.21-0.31$ \\
\hline
\end{tabular}

* Orbital phases were derived by the ephemerides of Skinner (1981; see text). The error on the orbital phase is about \pm 0.1 .

$\dagger$ This observation consists of two pointings separated by $\sim 20$ days and was not considered in the present analysis.

The data were analysed with the XANADU package: first the event files were taken from the ROSAT archive at the Max Planck Institute (München) and images were extracted, centered on the transient source position (Johnston et al. 1980). For each exposure the orbital phase was derived by using the $\mathrm{X}$-ray outbursts ephemeridis by Skinner (1981): the orbital period is $P_{\text {orb }}=16.6515 \pm 0.0005$ $\mathrm{d}$ and zero phase occurs at MJD $2443423.96 \pm 0.05$ (see Table 1). These uncertainties result in a typical error on the orbital phase of about \pm 0.1 .

The exposure maps were linearly interpolated and rescaled so to be overlaid on the images. Exposure corrected count rates or $3 \sigma$ upper limits were derived. Most of the observations have exposure times shorter than $1000 \mathrm{~s}$, so that different images, when close in time, were summed together in order to increase the signal to noise: 8 exposure corrected images were the final product of this proce- dure. A sliding-box detection algorithm was then applied to these summed images revealing A0538-66 seven times with a Poisson probability to be a background fluctuation smaller than $10^{-6}$ (see Table 2 ).

The highest count rates during a single observation were achieved on Jul $1992\left(\sim 0.03 \mathrm{c} \mathrm{s}^{-1}\right)$ and on Oct 1993 $\left(\sim 0.4 \mathrm{c} \mathrm{s}^{-1}\right)$. For these two observations the number of photons collected was sufficient to extract a spectrum.

The spectrum from the ROR 900543 (Oct 7, 1993) observation has been extracted from a circle of $\sim 4^{\prime}$ centered on the source and the background from a concentric annulus with inner and outer radii of 13' and 22', respectively. The 303 photons were rebinned into 11 energy channels in order to have at least 25 photons in each channel.

In Table 3 we report the results of the fitting for different models as well as the $90 \%$ confidence intervals. All single component spectral models provide a relative good fit to the data: a black body model with $T_{\mathrm{bb}}=0.2$ $\mathrm{keV}, R_{\mathrm{bb}}=7 \times 10^{6} \mathrm{~cm}$ and $N_{H}=3 \times 10^{20} \mathrm{~cm}^{-2} \mathrm{a}$ reduced $\chi^{2}=0.8$ (see Fig. 1); the $0.1-2.4 \mathrm{keV}$ flux amounts to $\sim 5 \times 10^{-12} \mathrm{erg} \mathrm{s}^{-1} \mathrm{~cm}^{-2}$, and, assuming a distance of $50 \mathrm{kpc}$, the luminosity to $\sim 10^{36} \mathrm{erg} \mathrm{s}^{-1}$. A bremsstrahlung model with a temperature of $T_{\mathrm{br}}=0.8$ $\mathrm{keV}$ and $N_{H}=7 \times 10^{20} \mathrm{~cm}^{-2}$ gives reduced $\chi^{2}=0.9$; the $0.1-2.4 \mathrm{keV}$ luminosity is slightly higher $\sim 3 \times 10^{36} \mathrm{erg} \mathrm{s}^{-1}$. In the case of a black body spectrum virtually all the observed flux falls inside the ROSAT PSPC energy range; for a bremsstrahlung spectrum the bolometric correction is about $30 \%$ for an energy range of $0.001-100 \mathrm{keV}$. A bolometric correction of about an order of magnitude is expected for a power-law model in the same energy range. If this is the case, a large fraction of the total luminosity would be emitted in the optical-UV band overwhelming the Be companion, so that the low energy cut off should be at lower energies and the bolometric correction smaller. Moreover, the power-law model provides a slightly worse fit to the ROSAT spectrum.

The source spectrum of the Jul 9, 1992 observation (ROR 400246) has been extracted from a circle of $\sim 3^{\prime}$ centered at the source position; the background has been estimated from a concentric annular region with inner and outer radius of 13' and 22', respectively. A smaller extraction radius than for ROR 900543 has been adopted in this case because the source was weaker and the background stronger. The 333 photons were rebinned into 12 energy channels in order to have at least 25 photons in each channel.

The spectrum is rather poor (see Table 3) and can be fitted by a black body with $T_{\mathrm{bb}}=0.3 \mathrm{keV}, R_{\mathrm{bb}} \lesssim 7 \times$ $10^{6} \mathrm{~cm}$ and $N_{H}=10^{21} \mathrm{~cm}^{-2}$ (reduced $\chi^{2}=1.0$ ); the 0.1-2.4 keV flux amounts to $\sim 4 \times 10^{-13} \mathrm{erg} \mathrm{s}^{-1} \mathrm{~cm}^{-2}$ and the luminosity to $\sim 10^{35} \mathrm{erg} \mathrm{s}^{-1}$. A bremsstrahlung spectrum with $T_{\mathrm{br}}=1.0 \mathrm{keV}$ and a $N_{H} \sim 3 \times 10^{21} \mathrm{~cm}^{-2}$ (reduced $\chi^{2}=1.0$ ); the $0.1-2.4 \mathrm{keV}$ luminosity is $\sim 3 \times$ $10^{35} \mathrm{erg} \mathrm{s}^{-1}$. The same bolometric corrections as before should be applied. 
Table 2. Summary of detections and upper limits for A0538-66.

$\begin{array}{cccccc}\text { Date } & \text { Time } & \text { Orbital } & \text { Count rate } & \text { Poisson } & \begin{array}{c}\text { Luminosity } \\ (\mathrm{s})\end{array} \\ \text { Phase } & 14521 & 0.62-0.65 & (3.3 \pm 0.3) \times 10^{-2} & <10^{-15} & \left.0.1-2.4 \mathrm{keV}^{-1} \mathrm{erg} \mathrm{s}^{-1}\right) \\ \text { Jul 9, 1992 } & 4082 & 0.36-0.43 & (9.1 \pm 2.2) \times 10^{-3} & 2 \times 10^{-8} & 3 \times 10^{35} \\ \text { Jul 1993* } & 1093 & 0.94 & (3.9 \pm 0.2) \times 10^{-1} & <10^{-15} & 1 \times 10^{36} \\ \text { Oct 7, 1993 } & 1244 & 0.61 & (1.6 \pm 0.4) \times 10^{-2} & 4 \times 10^{-7} & 6 \times 10^{34} \\ \text { Nov 4, 1993 } & 8749 & 0.07-0.57 & (2.0 \pm 0.5) \times 10^{-2} & 4 \times 10^{-13} & 7 \times 10^{34} \\ \text { Nov-Dec 1993*, } \dagger & 2002 & 0.46 & \lesssim 3.5 \times 10^{-2} & 1 \times 10^{-3} & \lesssim 1 \times 10^{35} \\ \text { Jun 1994* } & 5272 & 0.21-0.31 & (1.1 \pm 0.2) \times 10^{-2} & <10^{-15} & 4 \times 10^{34}\end{array}$

The upper limit to the count rate is at a level of $3 \sigma$. The luminosities were derived assuming a count rate to flux conversion of $1 \mathrm{c} \mathrm{s}^{-1}=1.2 \times 10^{-11} \mathrm{erg} \mathrm{s}^{-1} \mathrm{~cm}^{-2}$ based on spectral fits (black body model; in the case of a bremsstrahlung model the luminosities increase by a factor of $\sim 2$ ).

* Summed images.

$\dagger$ The major contributions to the detection do not come from the pointings near periastron.

Table 3. Summary of spectral fits for A0538-66.

\begin{tabular}{|c|c|c|c|}
\hline \multicolumn{4}{|c|}{ ROR 900543 (Oct 7, 1993) } \\
\hline Model & $\begin{array}{l}\text { Column density } \\
\qquad\left(10^{20} \mathrm{~cm}^{-2}\right)\end{array}$ & Parameter & Red. $\chi^{2}$ \\
\hline Black body $^{\dagger}$ & $3.3_{-2.1}^{+4.9}$ & $T_{\mathrm{bb}}=0.22_{-0.04}^{+0.07} \mathrm{keV}$ & 0.8 \\
\hline Bremsstrahlung & $7.5_{-2.7}^{+7.3}$ & $T_{\mathrm{br}}=0.81_{-0.37}^{+2.01} \mathrm{keV}$ & 0.9 \\
\hline Power law & $9.4_{-4.0}^{+12.3}$ & $\alpha=2.5_{-0.9}^{+1.2}$ & 1.0 \\
\hline Raymond-Smith & $1.9_{-1.8}^{+1.5}$ & $T_{\mathrm{RS}}=0.85_{-0.15}^{+0.25} \mathrm{keV}$ & 2.7 \\
\hline Disk Black body & $5.9_{-2.3}^{+5.9}$ & $T_{\mathrm{dd}}=0.33_{-0.09}^{+0.21} \mathrm{keV}$ & 0.9 \\
\hline \multicolumn{4}{|c|}{ ROR 400246 (Jul 9, 1992) } \\
\hline Model & $\begin{array}{l}\text { Column density } \\
\qquad\left(10^{21} \mathrm{~cm}^{-2}\right)\end{array}$ & Parameter & Red. $\chi^{2}$ \\
\hline Black body $^{\dagger}$ & $1.3_{-0.9}^{+9.9}$ & $T_{\mathrm{bb}}=0.31^{+0.34} \mathrm{keV}$ & 1.0 \\
\hline Bremsstrahlung & $2.8-0.7$ & $T_{\mathrm{br}}=0.98_{-0.82 \mathrm{keV}}$ & 1.0 \\
\hline Power law & 4.0 & $\alpha=3.0_{-2.9}$ & 1.0 \\
\hline Raymond-Smith & $15.1_{-7.1}^{+4.5}$ & $T_{\mathrm{RS}}=0.23_{-0.23}^{+0.03} \mathrm{keV}$ & 1.4 \\
\hline Disk Black body & $2.3_{-0.7}^{+10.7}$ & $T_{\mathrm{dd}}=0.42_{-0.42}^{+2.06} \mathrm{keV}$ & 1.0 \\
\hline
\end{tabular}

$\dagger$ The black body radius for the Oct 1993 observation is $R_{\mathrm{bb}}=6.9_{-2.1}^{+3.0} \times 10^{6} \mathrm{~cm}$; for the Jul 1992 the black body radius has large uncertainties, we derive $R_{\mathrm{bb}} \lesssim 7.3 \times 10^{6} \mathrm{~cm}$.

A careful analysis at the neutron star spin period was carried out. The upper limit on the $\mathrm{X}$-ray luminosity derived by Long et al. (1981) informs us that if matter acts to spin up the neutron star (and no outbursts occurred) the spin period will not change significantly. On the contrary, if during quiescence the system is in the propeller regime (see below) large spin downs are predicted: by taking the strongest version of the propeller regime (cf. Wang \& Robertson 1981), we derive that the spin period of A0538-66 should not exceed $75 \mathrm{~ms}$. We take the same PSPC counts used for the spectral analysis, after correcting their arrival times to the solar system barycenter. No significant periodicities were found in the range $68-75 \mathrm{~ms}$ providing a $3 \sigma$ upper limit of $\sim 85 \%$ on the amplitude of a sinusoidal $\mathrm{X}$-ray modulation. However, the pulsed component amplitude of A0538-66 detected by the Einstein MPC was of $\sim 25 \%$ (Skinner 1982). 

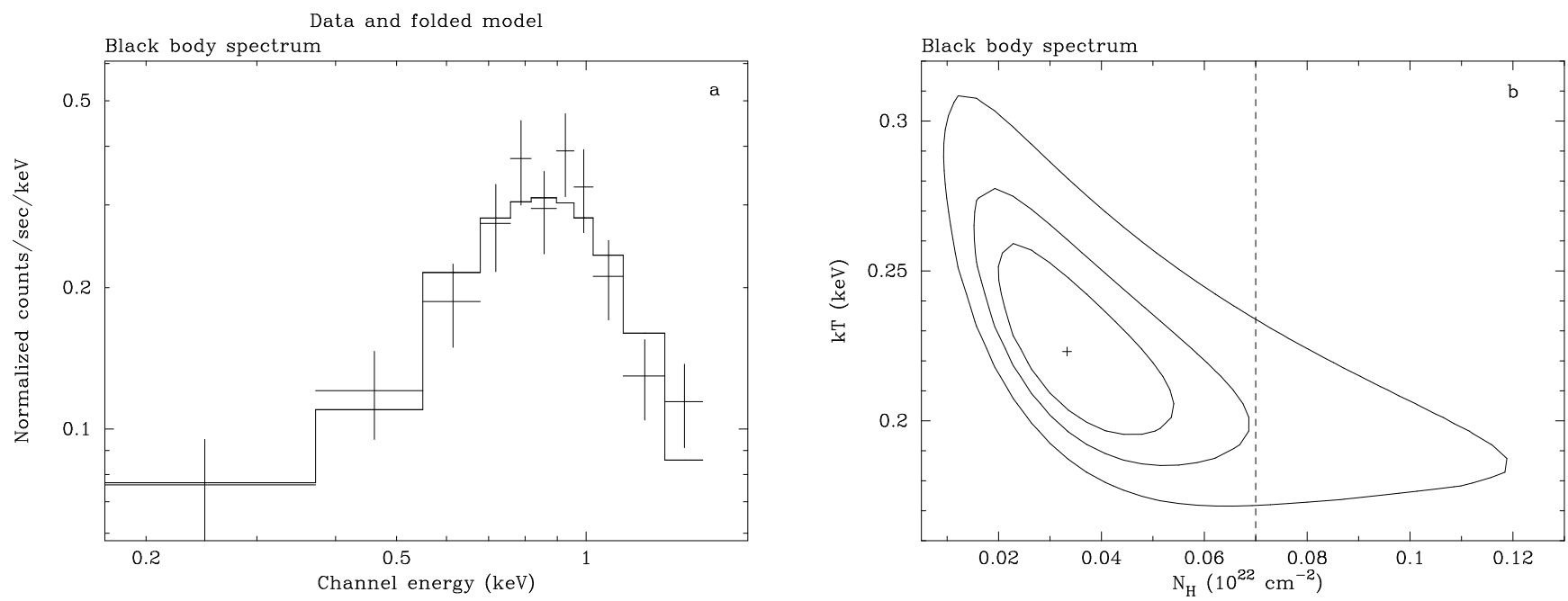

Fig. 1. In panel $a$ we report the spectrum of A0538-66 during the Oct, 71993 observation. The best fit model is provided by a black body spectrum. In panel $b$ the confidence contours (1, 2 and $3 \sigma$ ) for the black body model are reported. The cross signs the best fit model. The dashed line marks the mean galactic column density towards the Large Magellanic Cloud.

\section{Accretion in the propeller regime}

A0538-66 is a transient $\mathrm{X}$-ray source, the outburst of which likely derive from an enhanced emission of its Be companion star (hard X-ray transients). In the previous section we presented the first detection of A0538-66 during quiescence. The detected luminosity could in principle derive from different mechanisms (cf. Stella et al. 1994), its emission level is however sufficiently high not to be accounted by the Be companion star emission (which should emit $\sim 10^{32} \mathrm{erg} \mathrm{s}^{-1}$ at most; Meurs et al. 1992), nor by the emission of the underlying neutron star either due to cooling or by non-thermal processes. The most likely explanation is that the observed luminosity derives from accretion. Two different regimes are possible in this case (e.g. Illarionov \& Sunyaev 1975). The motion of the matter falling in the potential well of a neutron star becomes dominated by the rapidly increasing magnetic pressure at the magnetospheric radius, $r_{\mathrm{m}}$. At smaller radii the accretion flow follows the magnetic field lines and is enforced to corotate with the neutron star. If the magnetospheric radius lies inside the corotation radius $\left(r_{\text {cor }}\right)$, i.e. the radius at which a test particle in Keplerian orbit would corotate with the neutron star, the accreting matter can proceed down to the neutron star surface.

Due to the scaling of $r_{\mathrm{m}}$ with the accretion rate a minimum value, $\dot{M}_{\min }$, exists below which centrifugal acceler- ation is strong enough to expels the infalling matter, preventing the accretion. This limiting accretion rate $\dot{M}_{\text {min }}$ corresponds to a minimum accretion luminosity down to the neutron star surface of

$$
\begin{aligned}
L_{\min } & =\frac{G M \dot{M}_{\min }}{R} \\
& =8 \times 10^{38} \mu_{29}^{2} M_{1.4}^{-2 / 3} P_{69 \mathrm{~ms}}^{-7 / 3} R_{6}^{-1} \mathrm{erg} \mathrm{s}^{-1},
\end{aligned}
$$

where $P_{69} \mathrm{~ms}$ is the neutron star spin period in units of 69 ms and $M_{1.4}$ its mass in units of $1.4 M_{\odot}$. The neutron star magnetic moment $\mu=B R^{3} / 2$ is in units of $\mu=10^{29} \mu_{29}$ $\mathrm{G} \mathrm{cm}^{3}$, being $R=10^{6} R_{6} \mathrm{~cm}$ the neutron star radius and $B$ the surface magnetic field (e.g. Stella et al. 1994).

The high X-ray luminosity $\left(\sim 10^{39} \mathrm{erg} \mathrm{s}^{-1}\right.$; Skinner et al. 1980) and the presence of pulsations testify that during the bright outbursts the centrifugal barrier is open and accretion onto the neutron star surface takes place. As noted by Skinner et al. (1982), the lower range of X-ray luminosities observed during bright outbursts implies an upper limit of $\mu_{29} \lesssim 1$. On the other hand, the presence of X-ray pulsations indicates that a small magnetosphere is still present (i.e. $r_{\mathrm{m}}>R$ ); this implies $\mu_{29} \gtrsim 3 \times 10^{-3}$.

Below the minimum luminosity $L_{\min }$, the accretion flow is halted at the magnetospheric boundary, which behaves like a closed barrier. Entering the propeller regime 
a drop in the accretion-induced luminosity is expected to occur down to

$L_{\mathrm{cor}}=\frac{G M \dot{M}_{\mathrm{min}}}{r_{\mathrm{cor}}} \sim 3 \times 10^{37} \mu_{29}^{2} M_{1.4}^{-1} P_{69 \mathrm{~ms}}^{-3} \mathrm{erg} \mathrm{s}^{-1}$

which is the maximum allowed luminosity in this regime; for lower accretion rates the emitted luminosity scales as $\left(\dot{M} / \dot{M}_{\min }\right)^{9 / 7}$ (for more details see Campana et al. 1995; Corbet 1996).

If the minimum detected X-ray luminosity $(\sim 5 \times$ $10^{34} \mathrm{erg} \mathrm{s}^{-1}$, for a black body model) derives from accretion onto the neutron star surface, we have from eq. 1 an upper limit to the magnetic moment of $\mu_{29} \lesssim 8 \times 10^{-3}$. This field is likely too low to explain the observed pulsations during outburst. On the other hand, no stringent limitations on the magnetic moment exist if the accretion matter is stopped at the magnetospheric radius by the centrifugal barrier (one has to require $\mu_{29} \lesssim 10$ so that a radio pulsar cannot turn on; see Campana et al. 1995).

\section{Conclusions}

In Campana et al. (1995) we suggested that the lowluminosity ( $\lesssim 10^{38} \mathrm{erg} \mathrm{s}^{-1}$ ) and soft outbursts of A053866 (cf. Mavromatakis \& Haberl 1993) could be powered by accretion onto the neutron star magnetosphere, whereas during the outbursts with harder spectra and $L \gtrsim 10^{38} \mathrm{erg} \mathrm{s}^{-1}$ (when also the $69 \mathrm{~ms}$ pulsations were detected) the centrifugal barrier is open and the accretion flow can proceed down to the neutron star surface. This interpretation implies $\mu_{29} \sim 1$.

In this paper we report several detections of A0538-66 during quiescence. The $0.1-2.4 \mathrm{keV}$ luminosity is almost constant and in the range $3-10 \times 10^{34} \mathrm{erg} \mathrm{s}^{-1}$. Previous upper limits obtained with Einstein IPC were of $\sim 5 \times$ $10^{35} \mathrm{erg} \mathrm{s}^{-1}$ in the range $0.15-4.5 \mathrm{keV}$ (Long et al. 1981). On Oct 7, 1993 the PSPC flux increased by about an order of magnitude. This event took place at the same orbital phase as the one reported by Corbet et al. (1995) and it is likely to occur at the periastron passage (Skinner 1981). These events are also similar to the weak outbursts reported by Mavromatakis \& Haberl (1993).

The quiescent X-ray luminosity can hardly derive from accretion onto the neutron star surface because the required magnetic field is likely too low (see above). A better interpretation is that matter is stopped at the magnetospheric boundary by the centrifugal barrier, releasing gravitational energy up to $r_{\mathrm{m}}$. Therefore, A0538-66 is likely the first object detected in this propeller regime.

For this source the $\mathrm{X}$-ray spectrum is also available. Thermal models provide almost the same temperature during the weak outburst (both the one reported in this paper and the two discovered during the ROSAT all-sky survey) and in quiescence with $T_{\mathrm{bb}} \sim 0.2 \mathrm{keV}$.

The black body radius can be determined only for the observation with the highest count rate and, on that oc- casion, it is much larger than the neutron star radius and consistent with the magnetospheric radius: $R_{\mathrm{bb}} \gtrsim 5 \times 10^{6}$ $\mathrm{cm}$. Thermal emission from a standard disk "truncated" at the magnetospheric boundary may explain the softness of the spectrum and the large black body radius observed in A0538-66 during quiescence.

The accumulation of material outside the magnetospheric boundary has also been proposed to explain the soft thermal spectrum observed with the MPC onboard Einstein, during strong outburst (Ponman et al. 1984). Thermal emission from such "shells" has also been invoked for Her X-1 (McCray \& Lamb 1976) and SMC X-1 (Bunner \& Sanders 1979). During strong outbursts matter can fall down to the neutron star surface heating this shell $\left(T_{\mathrm{bb}} \sim 2 \mathrm{keV}\right)$; on the contrary during weak outbursts or in quiescence matter is likely stopped at $r_{\mathrm{m}}$. The lack of the underlying hard $\mathrm{X}$-ray spectrum results in a much smaller temperature $T_{\mathrm{bb}} \sim 0.2 \mathrm{keV}$, as testified by the lack of significant differences in the PSPC spectra during quiescence or weak outbursts.

Observations with the $A S C A$ and $S A X$ satellites could better constrain the emission properties of A0538-66 during quiescence.

Acknowledgements. I thank D. Lazzati, G.L. Israel, L. Stella and S. Mereghetti for helpful discussions. This work was partially supported by ASI.

\section{References}

Bunner A.N. \& Sanders W.T., 1979, ApJ, 228, L19

Campana S., Colpi M., Mereghetti S. \& Stella L., 1995, A\&A, 297,385

Corbet R.H.D., Smale A., Charles P. \& Southwell K., 1995, IAUC No. 6136

Corbet R.H.D., 1996, ApJ, 457, L31

Illarionov A.F., \& Sunyaev R.A., 1975, A\&A, 39, 185

Johnston M.D., Griffiths R.E. \& Ward M.J., 1980, Nature, 285, 26

Long K.S., Helfand D.J. \& Grabelsky D.A., 1981, ApJ, 248, 925

Mavromatakis F. \& Haberl F., 1993 A\&A, 274, 304

McCray R. \& Lamb F.K., 1976, ApJ, 204, L115

Meurs E.J.A. et al., 1992, A\&A, 265, L41

Ponman T.J., Skinner G.K. \& Bedford D.K., 1984, MNRAS, 207,621

Skinner G.K. et al., 1980, ApJ, 240, 619

Skinner G.K., 1981, Sp. Sci. Rev., 30, 441

Skinner G.K. et al., 1982, Nature, 297, 568

Stella L., Campana S., Colpi M., Mereghetti S. \& Tavani M., 1994, ApJ, 423, L47

Stella L., White N.E. \& Rosner R., 1986, ApJ, 308, 669

Wang Y.-M. \& Robertson J.A., 1981, A\&A, 151, 361

White N.E. \& Carpenter G.F., MNRAS, 183, 11P

This article was processed by the author using Springer-Verlag $\mathrm{LAT}_{\mathrm{E}} \mathrm{X}$ A\&A style file $L-A A$ version 3 . 